\title{
A Method for Distributed Generator Dispatch Strategy in Distribution Network
}

\author{
Md. Asaduz-Zaman ${ }^{1}$, Md. Habibur Rahaman ${ }^{2}$, Md. Selim Reza ${ }^{3}$ and Md. Mafizul Islam ${ }^{4}$ \\ 1. Dept. of Textile Machinery Design and Maintenance, Bangladesh University of Textiles, Dhaka 1208, Bangladesh \\ 2. Dept. of ECE, Rajshahi University of Engineering \& Technology, Rajshahi 6204, Bangladesh \\ 3. Dept. of EEE, Bangladesh Army University of Engineering \& Technology, Natore 6400, Bangladesh \\ 4. Dept. of Textile Machinery Design and Maintenance, Bangladesh University of Textiles, Dhaka 1208, Bangladesh
}

\begin{abstract}
Since a load of power system changes continuously, the generation also adjusted for supply-demand balance purpose. If there exist more distributed generators in the distribution network, the dispatch strategy becomes more crucial. The possibility of having numerous controllable microgrids, diesel generator (DG) units and loads for microgrids (MGs) system requires an efficient dispatch strategy in order to balance supply demand for reducing the total cost of the integrated system. In this paper, a method for the dispatch of the distributed generator in distributed power systems has been proposed. The dispatch strategy is such that it keeps a flat voltage profile, reduces the network losses, increases the maximum loading and voltage security margin of the system. The procedure is based on the analysis of continuous power flow. The method is executed on a 34-bus test system. The MATLAB based PSAT packages are used for simulation purpose.
\end{abstract}

Key words: Distributed generator, dispatch, distributed network, active loss, reactive loss, maximum loading parameter, continuation power flow.

\section{Introduction}

Presently the demand for distributed generation (DG) increases rapidly due to environmental issues and transmission loss reduction purpose. DG also increases the reliability of the system. DGs are particularly advantageous as these are deployed in close proximity to the demand, enabling higher efficiency [1].

If the location or capacity of DG changes, then both the voltage and current the distribution in distribution network change. This influences the voltage profile and power loss of the distribution network. The location and injecting capacity are not properly designed, the power losses would increase and harm the stability of the system [2]. The adverse effects of installing DG are well known [3-5]. In distribution network there may exist many sizes of the distributed

Corresponding author: Md. Asaduz-Zaman, B.Sc. in EEE, assistant professor, research fields: smart grid, microgrid etc. generator and also their location may be different. If load changes, then the DG output power also changes for matching supply-demand balance. For this purpose dispatch of DG is necessary. The proper dispatch brings a positive influence to the distributed grid. The stable voltage can be maintained and the power losses can be reduced.

The dispatch strategy of DG units in the distribution network is one of the major challenges. There are some technical issues like the voltages on the buses, the system losses, and security margin etc. The economical assessments include emissions, operating costs etc. [6-9]. Though different literature pays attention to dispatch of DG which mainly directed to economic benefit [10-13]. The placement of DGs is also important to reduce voltage fluctuation or collapse.

In Ref. [14] the solution of DGs placement problem has been discussed with all possible solutions that are previously published. It is very well-known that in a 
distributed microgrid DGs supply real and reactive power to the grid. As the real power is the useful power but the reactive power should be controlled for better performance. In Ref. [15] a new method called as segmented-time reactive power control method has been proposed to solve segmented-time reconfiguration problem by using hybrid particle swarm optimization (HPSO) method. But problem is that solvers CPLEX and CONOPT are used to solve complicated main and slave mathematical problems respectively. In Ref. [16] an IEEE 14-bus distribution system has been simulated to distribute electric power for charging electric vehicles by using quadratic rotation cone programming with multi-state probability model instead of HPSO method. It showed that the power distribution performance is excellent but network installation cost, generation cost, line efficiency had not been described.

It is found from previous studies that the energy has been dispatched from centralized controlling by using mixed integer linear problem (MILP), but due to thermal unit commitment problem, this method has suffered optimization problem [17]. To overcome this issue, the load side power management system has been considered in Ref. [18]. A mixed integer non-linear programming (MINLP) method has been briefly discussed in Ref. [19] both for grid-connected mode and islanded mode. Although this method is suitable for energy dispatch in the distributed network at all constraints the non-linear methodical model is very complicated. It is observed that a few number of research papers have been found about to regain voltage profile stability with lowest generation cost.

For dispatching DG in the distribution network the technical issues should be verified. Flat voltage profiles should be ensured and voltage security margin has to increase for reliable power supply to the consumer. Since the main objective of the power network is to make the voltage per unit to unity value, it is one of the most important objectives for power network directors are to have a flat voltage profile [20]. If the value of voltage security margin (VSM) is low, then the system will be weak and at the proximity of voltage collapse [21].

In this paper, a method for distributed generator dispatch strategy in the distribution network is proposed. The dispatch procedure is based on continuation power flow analysis. The objective is to keep the flat voltage profile, minimize losses, increase maximum loading and voltage security margin. The remainder of this paper is organized as follows. The next section briefly describes continuation power flow method. Section 3 elaborates on a proposed algorithm for DG dispatch in distribution network which seeks objective. Also, some index for the performance of DG is defined. Simulation runs are carried out on the well-known 34-bus radial distribution network in Section 4 and the result is analyzed. Finally, Section 5 concludes this paper.

\section{Continuation Power Flow}

The determination of maximum loading is one of the most important problems in voltage stability analysis. Conventional power flow programs (PFPs) are prone to convergence problems when operating near the stability limit [22]. This type of problem can be overcome by continuation power flow (CPF) method so that they remain well-conditioned at all possible loading conditions. A continuous power flow uses a successive solution to compute the voltage profile up to a collapse point or bifurcation point where the Jacobian matrix of linearized power flow Eq. (1) becomes singular [14].

$$
\left[\begin{array}{c}
\Delta P \\
\Delta Q
\end{array}\right]=\left[\begin{array}{ll}
J_{P \theta} & J_{P V} \\
J_{Q \theta} & J_{Q V}
\end{array}\right]\left[\begin{array}{l}
\Delta \theta \\
\Delta V
\end{array}\right]
$$

The reformulated power-flow equations, with provision for increasing generation as the load is increased, may be expressed as [22],

$$
F(\theta, V, \lambda)=0
$$

where $\lambda$ is the loader parameter and a scalar variable 
which multiplies generator and load directions as follows [23].

$$
\begin{aligned}
& P_{G}=P_{G 0}+\left(\lambda+\gamma k_{G}\right) P_{s} \\
& P_{L}=P_{L 0}+\lambda P_{D} \\
& Q_{L}=Q_{L 0}+\lambda Q_{D}
\end{aligned}
$$

where $P_{G 0}, P_{L 0}$ and $Q_{L 0}$ are the base case generator and load powers, whereas $P_{S}, P_{D}$ and $Q_{D}$ are the generator and load power directions. If power directions data are not defined, the base case powers are used as load directions and Eq. (1) becomes

$$
\begin{aligned}
& P_{G}=\left(\lambda+\gamma k_{G}\right) P_{G 0} \\
& P_{L}=\lambda P_{L 0} \\
& Q_{L}=\lambda Q_{L 0}
\end{aligned}
$$

The distributed slack bus variable $\left(k_{G}\right)$ and the generator participation coefficients $(\gamma)$ are optional. The CPF analysis uses an iterative process involving predictor and corrector steps [22].

Predictor step: In the predictor step, a linear approximation is used to estimate the next solution for a change in one of the state variables (i.e. $\theta, V, \lambda$ ). Actually the predictor step is used to indicate a direction to move and the tangent vector can be used for this purpose. Taking the derivative of Eq. (2), we get the following set of linear equations [22].

$$
\left[\begin{array}{lll}
F_{\theta} & F_{V} & F_{\lambda}
\end{array}\right]\left[\begin{array}{l}
d \theta \\
d V \\
d \lambda
\end{array}\right]=[0]
$$

Eq. (5) can be solved by setting one of the tangent vectors to +1 or -1 . This component is called a continuation parameter.

$$
\left[\begin{array}{ccc}
F_{\theta} & F_{V} & F_{\lambda} \\
e_{k} &
\end{array}\right]\left[\begin{array}{l}
d \theta \\
d V \\
d \lambda
\end{array}\right]=\left[\begin{array}{l}
0 \\
\pm 1
\end{array}\right]
$$

where $e_{k}= \pm 1$, and its sign determines the increase or the decrease of $\lambda$.

Corrector step: In the corrector step, the original set of equations $F(\theta, V, \lambda)=0$ is augmented by one equation that specifies the state variables selected as the continuation parameter.

$$
\left[\begin{array}{c}
F(\theta, V, \lambda) \\
x_{k}-\eta
\end{array}\right]=[0]
$$

where $x_{k}$ is the state variable selected as the continuation parameter and $\eta$ is selected equal to the predicted value of $x_{k}$. Adding additional equation specifying $x_{k}$ makes the Jacobian matrix non-singular at the collapse point which is the maximum loading $\left(\lambda^{\max }\right)$. The perpendicular intersection or the local parameterization can be used to obtain the predictor step [24]. The appropriate selection of continuation parameter is important for the corrector step [22].

\section{Algorithm for DG Dispatch Strategy}

For diesel generator (DG) dispatch, it is necessary to define objective function for solving the problem. In this section, the problem is formulated and solved by using continuation power flow (CPF) method. The objective includes keeping a flat voltage profile, loss reduction and voltage security margin enhancement.

\subsection{DG Selection Process}

The algorithm for DG dispatch is shown in Fig. 1. According to algorithm after receiving data, maximum loading is determined by CPF method and power losses by power flow program (PFP). In this case, no DG is connected there and it is considered as the base case of the distribution network. This data will be used for calculating the performance indices for DG dispatch. After that, the maximum loading of the distribution network is determined by using continuation power flow method for each DG. A priority list of candidate DG for dispatch is generated based on maximum loading. The DG for which the distribution network has the highest loading is given the first priority and selected as the candidate. Now power flow program is executed with candidates DG and total losses are determined. If the system losses increase for this DG then the next highest loading DG from the priority list is selected as another candidate 


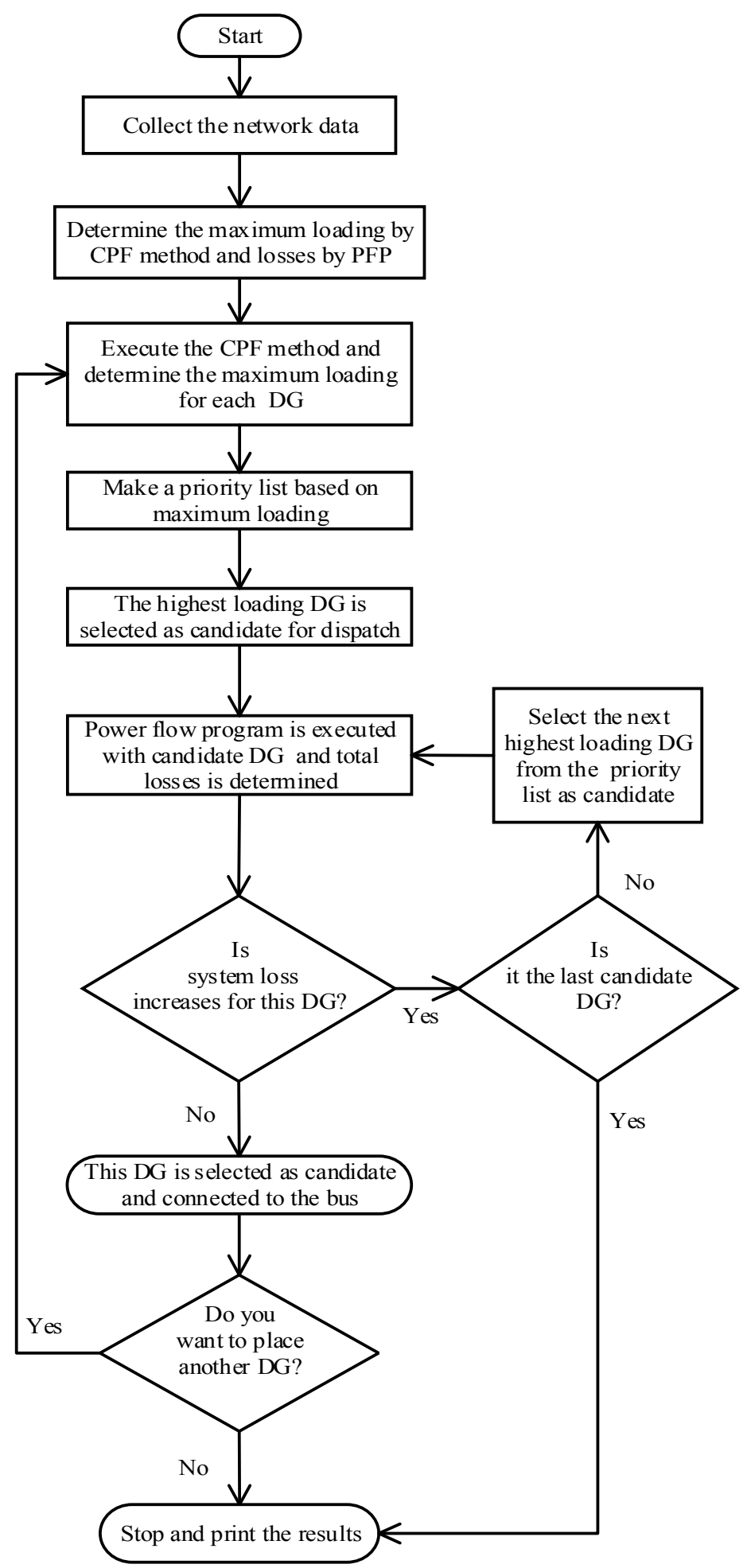

Fig. 1 Proposed algorithm for DG dispatch.

until the last one. Otherwise this DG is selected for dispatch if the system loss reduces. Then, this DG is installed. If more DGs are allowed to dispatch, the procedure will be repeated, taking into account the already selected DG/DGs stay connected to the distribution network. 


\subsection{DG Dispatch Indices}

Several indices are used to evaluate the performance of DG units on distribution networks. The active loss reduction, reactive loss reduction, voltage security margin and flat voltage profile are considered as the performance indicator for DG dispatch.

The active loss reduction (ALR) and reactive loss reduction (RLR) is calculated by the following formula [23].

$$
\begin{aligned}
& A L R=\frac{P_{\text {Loss }}-P_{\text {Loss }}^{D G}}{P_{\text {Loss }}} \times 100 \% \\
& R L R=\frac{Q_{\text {Loss }}-Q_{\text {Loss }}^{D G}}{Q_{\text {Loss }}} \times 100 \%
\end{aligned}
$$

where,

$P_{\text {Loss }}=$ Total active power losses without DG

$P_{\text {Loss }}^{D G}=$ Total active power losses with DG

$Q_{\text {Loss }}=$ Total reactive power losses without DG

$Q_{\text {Loss }}^{D G}=$ Total reactive power losses with DG

The higher values of $A L R$ and $R L R$ indicate better performance of DGs in loss reduction.

The voltage security margin (VSM) of the system is given by,

$$
V S M=\lambda^{\max }-\lambda^{0}
$$

where $\lambda^{\max }$ is the maximum loading and $\lambda^{0}$ is the reference loading which is considered to be 0 in this paper [22]. The higher the VSM, the more secure the system. The voltage profile is graphically evaluated.

\section{Simulation Result and Analysis}

The proposed method is applied to a 34-bus test system (see Appendix A and Fig. 8). For simulation purpose, it is considered that five DG units exist at Bus 11, Bus 21, Bus 26, Bus 29 and Bus 33. The capacity of each DGs is same and can deliver 2,000 $\mathrm{kW}$ active and 1,500 kvar reactive power. In each iteration of the distributed generation dispatch algorithm, one DG unit is connected. The results present the robustness of this method for the fast dispatch of DG. The bus voltage, active power loss, reactive power loss and loading parameter are expressed in per unit (p.u.).

The maximum loading parameter is determined by continuation power flow method. The active power loss and reactive power loss is determined by the power flow program for the base case where no DG units are connected to the distribution network. Fig. 2 shows the loading parameter vs. bus voltage profile at the candidate buses for the base case.

Now the algorithm is executed for the first time by connecting each DG individually to the network. The maximum loading up to the voltage collapse point is determined using continuation power flow method as shown in Fig. 3a. The DG priority list is created based on maximum loading. According to this, DG 26 is given the first priority, then DG 33, DG 11, DG 29 and DG 21 gradually. Now using the power flow program the active and reactive power losses are calculated for DG26 as shown in Fig. 3b. This loss is less than the base case losses. So DG 26 is now selected for dispatch. The losses for other DGs are also shown which is not part of the algorithm. It is observed that lowest loss occurs for DG 21 but its maximum loading is low and is not suitable for dispatch. Similarly, others result can be observed.

For dispatch purpose of another DG, again algorithm is executed where first selected DG 26 is always staying connected. The maximum loading for DGs determined is shown in Fig. 4a. The DG 29 is given the first priority for its highest maximum loading, then DG 11, DG 33 and DG 21. Also, active and reactive power losses are reduced for DG 29 are shown in Fig. 4b. As a result, DG 29 is selected for dispatch. Again it can be observed that the losses for DG 11 are reduced, but maximum loading is low than DG 29. So dispatch of DG 29 is the perfect decision.

Now it is the third iteration of the algorithm. Maximum loading for other DGs is determined where already selected DG 26 and DG 29 stay connected as 


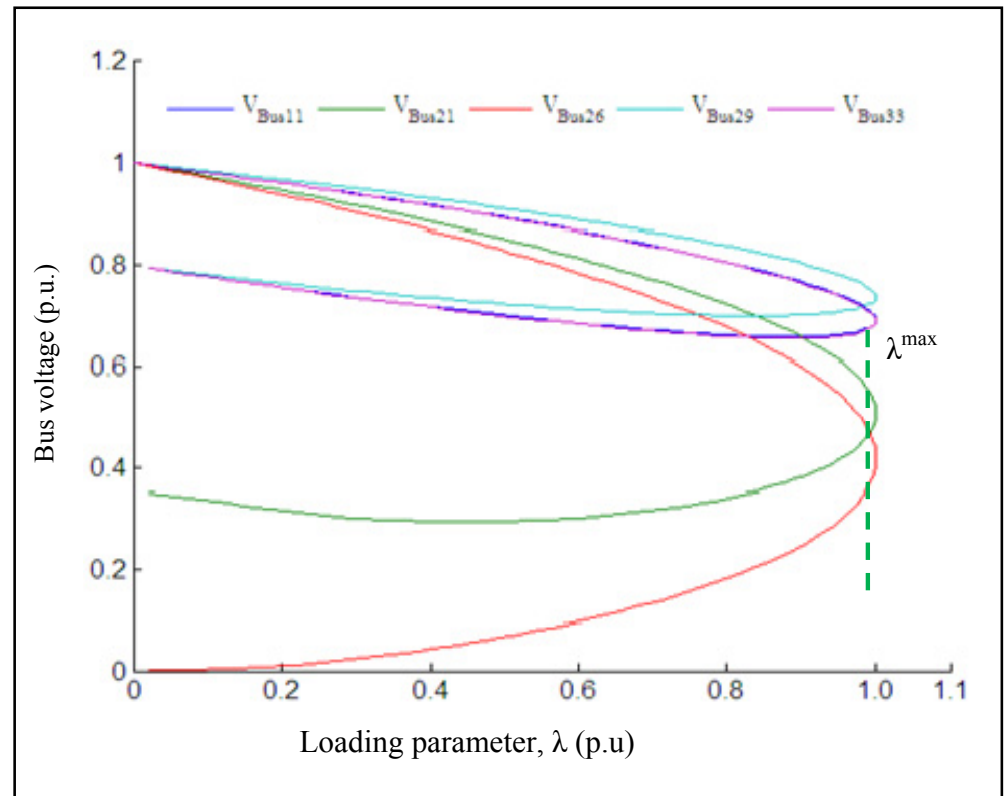

Fig. 2 Loading parameter vs. bus voltage.

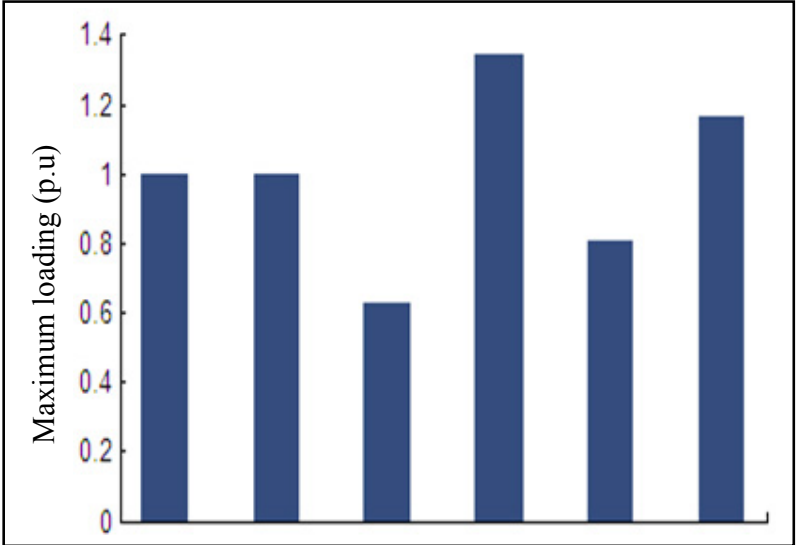

(a)

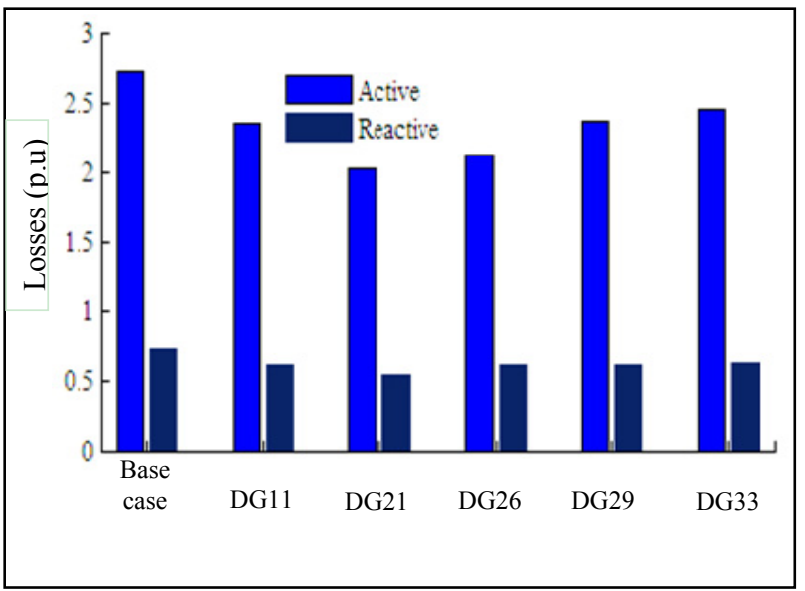

(b)

Fig. 3 (a) Maximum loading parameter and (b) total system losses.

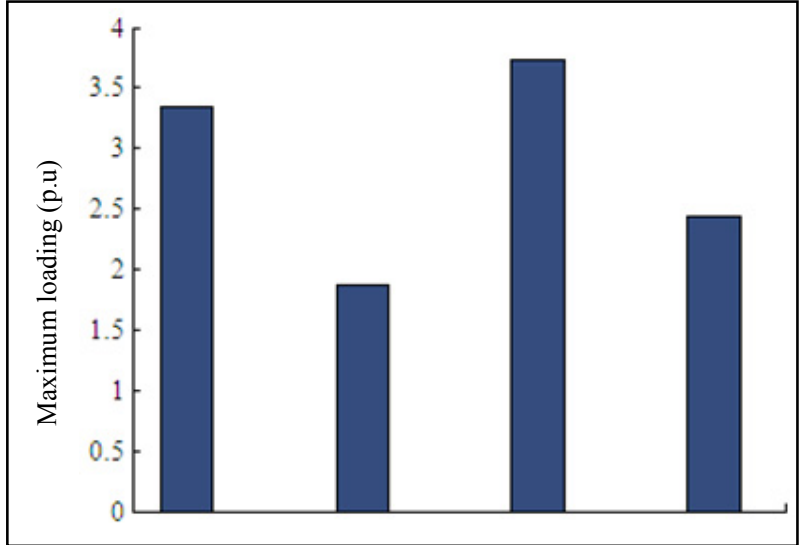

(a)

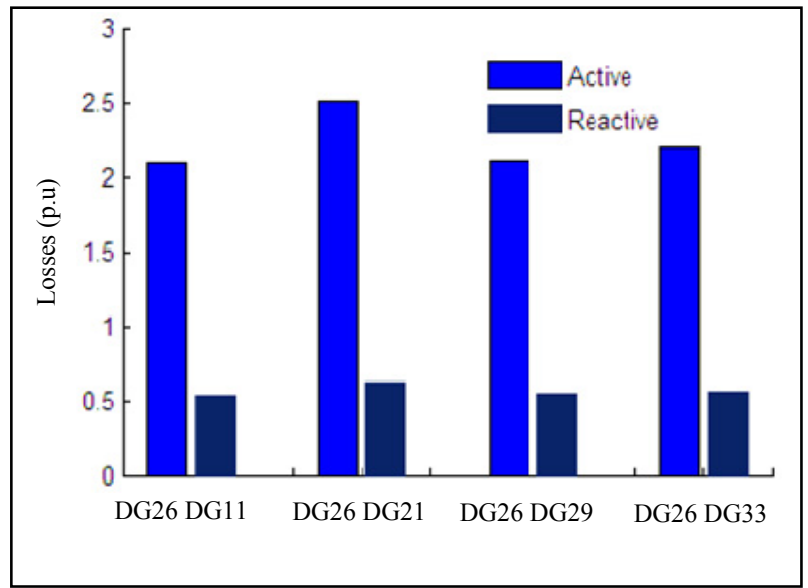

(b)

Fig.4 (a) Maximum loading parameter and (b) total system losses. 


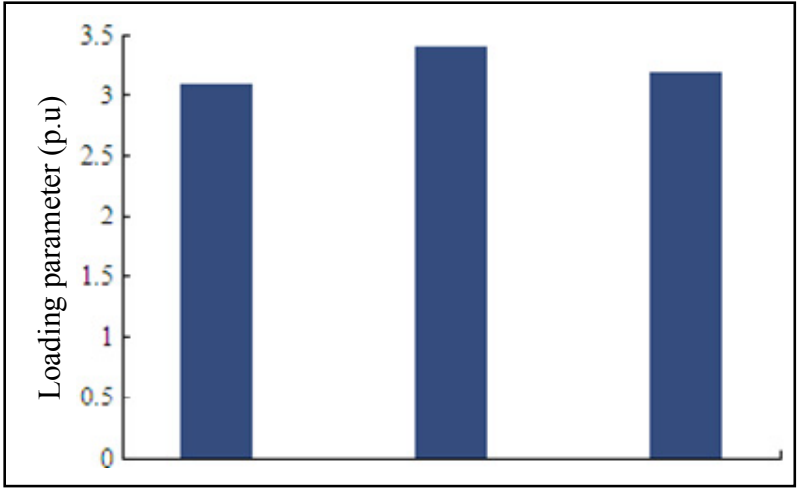

(a)

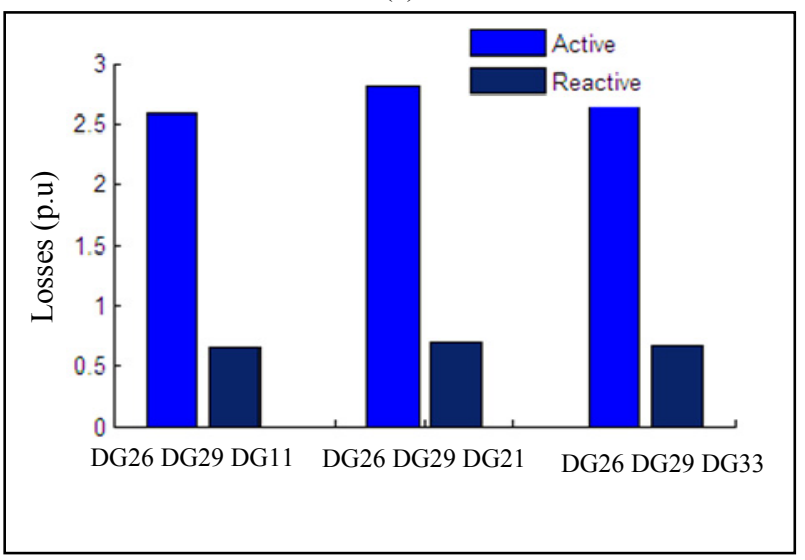

(b)

Fig.5 (a) Maximum loading parameter and (b) total system losses.

given in Fig. 5a. The priority list is as follows: DG 21, DG 11 and DG 33. The losses for DG 21 increase. Now losses for next priority DG 11 are determined. Again the system losses are high compared to the previous case. Similar results can be shown for DG 33 . As a result, no more DG units can be connected to the network for dispatch purpose as shown in Fig. 5b.

Now the DG dispatch indices are evaluated. A comparative list of active power loss reduction, reactive power loss reduction, and voltage security margin is given in Table 1. After DG dispatch the value of ALR, RLR and VSM is $28.92 \%, 36.70 \%$ and 3.73 respectively. The higher values of ALR, RLR, and VSM indicate the excellent performance of the proposed DG dispatch strategy. Also, Fig. 6 shows the voltage at the collapse point where the maximum loading occurs for three different scenarios. Similarly Fig. 7 shows the operating voltage after DG dispatch.
Table 1 Losses and voltage security margin comparison.

\begin{tabular}{llll}
\hline Scenarios & ALR (\%) & RLR (\%) & VSM \\
\hline No DG & --- & --- & 1.00 \\
DG26 & 21.81 & 31.93 & 1.35 \\
DG 26 \& DG 29 & 28.92 & 36.70 & 3.73 \\
\hline
\end{tabular}

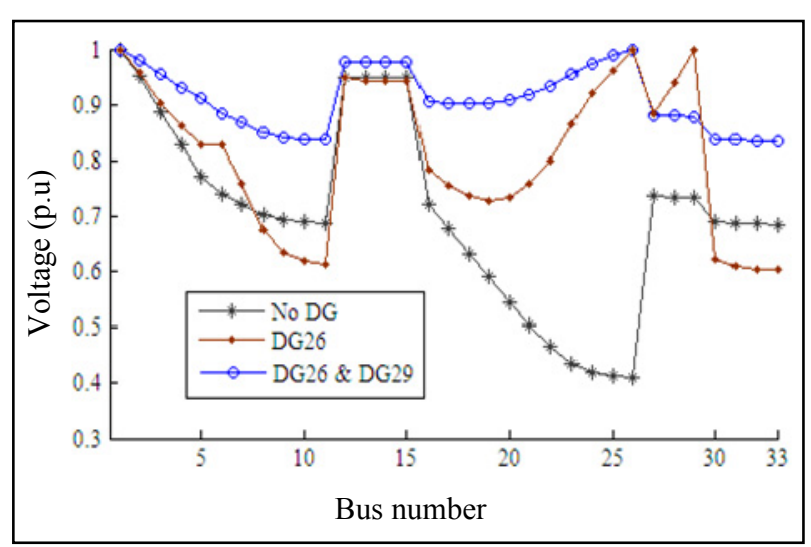

Fig.6 Voltage collapse profile curve for 34-bus system.

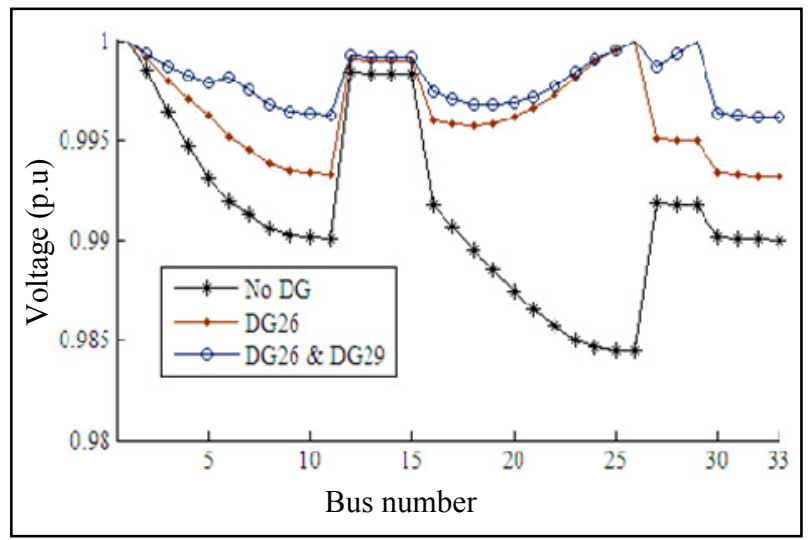

Fig. 7 Voltage profile curve for 34-bus system.

The voltage profile improves and becomes flattered compared to the base case when the distributed generator is dispatched according to our proposed method.

\section{Conclusion}

A method for distributed generator dispatch strategy in the distribution network is presented. Using this procedure the active and reactive power losses can be reduced. The voltage security margin is increased to a considerable amount. Also, the voltage profile is improved and becomes flatter. The method is applied to a 34-bus test system and various performance indexes are determined and compared. 
The results prove the robustness of the proposed method for the dispatch of DG units in the distribution system, though in this method only technical issues are considered. The dispatch strategy will be preferable if both technical and economic issues are included in the method.

\section{References}

[1] Srikantha, P., and Kundur, D. 2014. "Distributed Power Dispatch via Bifurcation Control." In Proceedings of Global Conference on Signal and Information Processing, Atlanta, GA, 3-5 December 2014, pp. 228-32.

[2] Bo, W., and Ka, L. 2011. "Analysis of the Distributed Generation System and the Influence on Power Loss." In Proceedings of Asia-Pacific Power and Energy Engineering Conference (APPEEC), Wuhan, 25-28 March 2011, pp. 1-4.

[3] Pecas Lopes, J. A. 2012. "Integration of Dispersed Generation on Distribution Networks-Impact Studies." In Proceedings of Power Engineering Society Winter Meeting, 2002, Vol. 1, pp. 323-8.

[4] Vovos, P. N., Kiprakis, A. E., Wallace, A. R., and Harrison, G. P. 2007. "Centralized and Distributed Voltage Control: Impact on Distributed Generation Penetration." IEEE Transactions on Power Systems 22 (1): 476-83.

[5] Abbott, S. R., Fox, B., and Morrow, D. J. 2014. "Sensitivity-Based Dispatch of DG for Voltage Control." In Proceedings of PES General Meeting, Conference \& Exposition, National Harbor, MD, 27-31 July 2014, pp. $1-5$.

[6] Kamalinia, S., Afsharnia, S., Khodayar, M. E., Rahimikian, A., and Sharbafi, M. A. 2007. "A Combination of MADM and Genetic Algorithm for Optimal DG Allocation in Power Systems." In Proceedings of 42nd International on Universities Power Engineering Conference, Brighton, 4-6 September 2007, pp. 1031-5.

[7] Asadi, A., Friuzabad, M. F., and Moeini-Aghtaie, M. 2014. "Techno-Economic Considerations on Distributed Generations Planning Studies in Power Distribution Systems." In Proceedings of 19th Conference on Electrical Power Distribution Networks, Tehran, 6-7 May 2014, pp. 82-7.

[8] Payyala, S. L., and Green, T. C. 2007. "An Estimation of Economic Benefit Values of DG." IEEE Power Engineering Society General Meeting, Tampa, FL, 24-28 June 2007, pp. 1-8.

[9] Biswas, S., Chatterjee, A., and Kumar Goswami, S. 2014. "An Artificial Bee Colony Based Optimal Placement and
Sizing of Distributed Generation." In Proceedings of International Conference on Control, Instrumentation, Energy and Communication, Calcutta, Jan. 31-Feb. 2, 2014, pp. 356-60.

[10] Gang, C., and Ning, F. E. 2015. "Distributed Optimal Dispatch of Multiple Networked Generators." In Proceedings of 27th Chinese Control and Decision Conference, Qingdao, China, 23-25 May 2015, pp. 5546-51.

[11] Gwo-Ching, L. 2010. "Using Chaotic Quantum Genetic Algorithm Solving Environmental Economic Dispatch of Smart Microgrid Containing Distributed Generation System Problems." In Proceedings of International Conference on Power System Technology, Hangzhou, 24-28 Oct. 2010, pp. 1-7.

[12] Gwo-Ching, L. 2013. "The Optimal Economic Dispatch of Smart Microgrid Including Distributed Generation.” In Proceedings of IEEE International Symposium on Next-Generation Electronics, Kaohsiung, 25-26 Feb. 2013, pp. 473-7.

[13] Gwo-Ching, L., and Jong-Ian, T. 2012. "Use a New Method to Solve the Economic Dispatch Problem of Smart Microgrid Including Distributed Generation." In Proceedings of Asia-Pacific Power and Energy Engineering Conference, Shanghai, 27-29 March 2012, pp. 1-4.

[14] Ettehadi, G., and Vaez-Zadeh, S. 2013. "Voltage Stability-Based DG Placement in Distribution Networks." IEEE Transactions on Power Delivery 28 (1): 171-8.

[15] Chen, S., Hu, W., and Chen, Z. 2015. "Comprehensive Cost Minimization in Distribution Networks Using Segmented-Time Feeder Reconfiguration and Reactive Power Control of Distributed Generators." IEEE Transections on Power Systems 31 (2): 983-93.

[16] Wu, J., Wu, Z., Wu, F., and Mao, X. 2017. “A Power Balancing Method of Distributed Generation and Electric Vehicle Charging for Minimizing Operation Cost of Distribution Systems with Uncertainties." Energy Science and Engineering 5 (3): 167-79.

[17] Korpas, M., and Holen, A. T. 2006. "Operation Planning of Hydrogen Storage Connected to Wind Power Operating in a Power Market." IEEE Trans. Energy Convers. 21 (3): 742-9.

[18] Shi, W., Member, S., Li, N., and Xie, X. 2014. "Optimal Residential Demand Response in Distribution Networks." IEEE J. Sel. Areas Commun. 32 (7): 1441-50.

[19] Zaree, N., and Vahidinasab, V. 2016. "An MILP Formulation for Centralized Energy Management Strategy of Microgrid." In Proceedings of IEEE Smart Grids Conference (SGC), 20-21 Dec. 2016, Iran, pp. 130-7.

[20] Banihashemi, F., Lesani, H., and Salar Makhzani, A. 
2013. “ Locating and Capacity Determination of Distributed Generations Using None-dominated Sorting Genetic Algorithm." In Proceedings of IEEE Grenoble Power Tech, Grenoble, 16-20 June 2013, pp. 1-8.

[21] Banerjee, S., Chattopadhyay, T. K., and Chanda, C. K. 2012. "Voltage Stability Margin of Distribution Networks for Composite Loads." In Proceedings of Annual IEEE
India Conference, Kochi, 7-9 December 2012, pp. 582-7.

[22] Kundur, P. 1994. Power System Stability and Control. New York, NY: McGraw-Hill.

[23] Hedayati, H., Nabaviniaki, S. A., and Akbarimajd, A. 2008. "A Method for Placement of DG Units in Distribution Networks." IEEE Transactions on Power Delivery 23 (3): 1620-8.

Appendix A

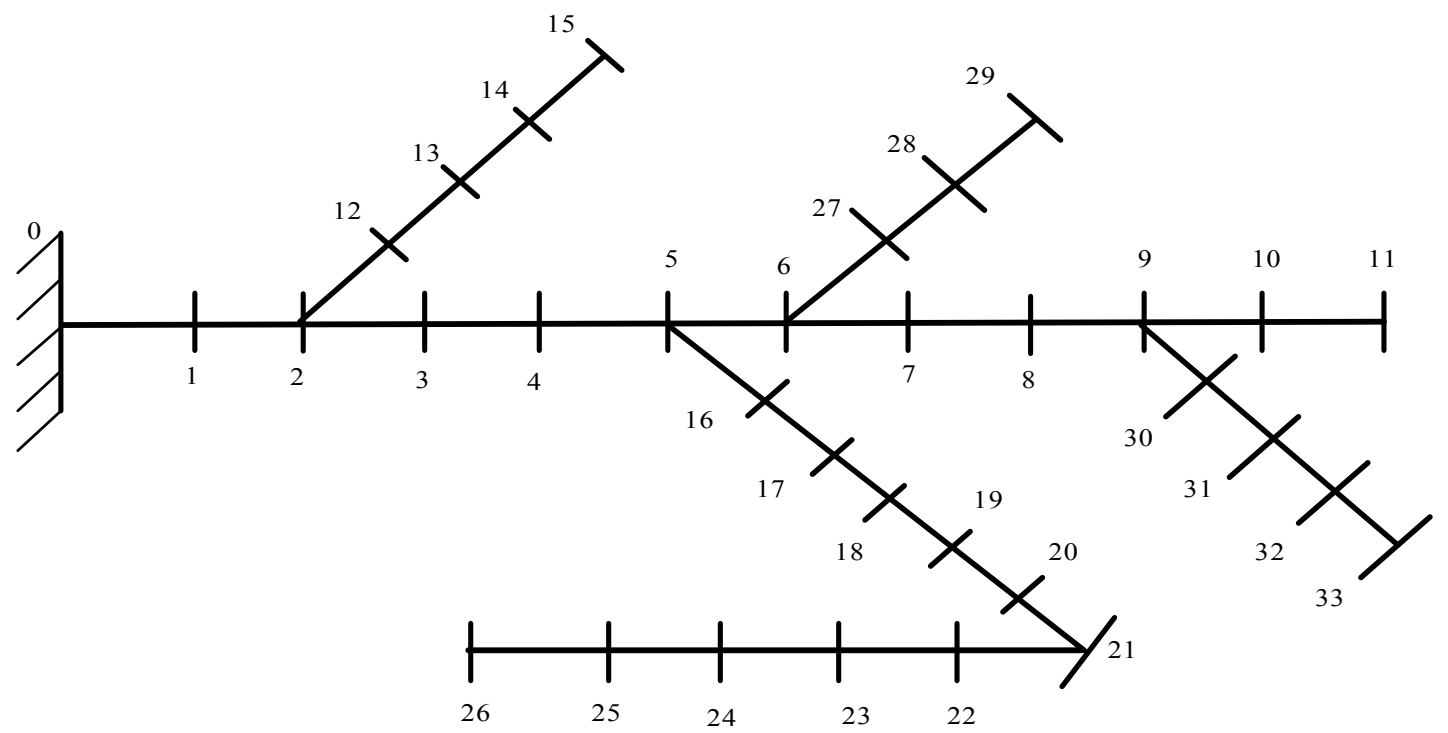

Fig. 8 34-Bus test radial network. 
Table 2 Load and distribution lines data.

\begin{tabular}{|c|c|c|c|c|c|}
\hline $\begin{array}{l}\mathrm{L} \\
(\mathrm{km})\end{array}$ & $\begin{array}{l}\mathrm{Q} \\
\text { (kvar) }\end{array}$ & $\begin{array}{l}\mathrm{Q} \\
(\mathrm{kW})\end{array}$ & $\begin{array}{l}\mathrm{X} \\
\text { (ohm/km) }\end{array}$ & $\begin{array}{l}\mathrm{R} \\
\text { (ohm/km) }\end{array}$ & Bus \\
\hline 0.6 & 142.5 & 230 & 0.08 & 0.195 & $0-1$ \\
\hline 0.55 & --- & --- & 0.08 & 0.195 & $1-2$ \\
\hline 0.55 & 142.5 & 230 & 0.083 & 0.299 & $2-3$ \\
\hline 0.5 & 142.5 & 230 & 0.083 & 0.299 & $3-4$ \\
\hline 0.5 & --- & --- & 0.083 & 0.299 & $4-5$ \\
\hline 0.6 & --- & --- & 0.09 & 0.524 & $5-6$ \\
\hline 0.4 & 142.5 & 230 & 0.09 & 0.524 & $6-7$ \\
\hline 0.6 & 142.5 & 230 & 0.09 & 0.524 & $7-8$ \\
\hline 0.4 & --- & --- & 0.09 & 0.524 & $8-9$ \\
\hline 0.25 & 142.5 & 230 & 0.09 & 0.524 & $9-10$ \\
\hline 0.2 & 84 & 137 & 0.09 & 0.524 & $10-11$ \\
\hline 0.3 & 45 & 72 & 0.09 & 0.524 & $11-12$ \\
\hline 0.4 & 45 & 72 & 0.09 & 0.524 & $12-13$ \\
\hline 0.2 & 45 & 72 & 0.09 & 0.524 & $13-14$ \\
\hline 0.1 & 7.5 & 13.5 & 0.09 & 0.524 & $14-15$ \\
\hline 0.6 & 142.5 & 230 & 0.083 & 0.299 & $15-16$ \\
\hline 0.55 & 142.5 & 230 & 0.083 & 0.299 & $16-17$ \\
\hline 0.55 & 142.5 & 230 & 0.086 & 0.378 & $17-18$ \\
\hline 0.5 & 142.5 & 230 & 0.086 & 0.378 & $18-19$ \\
\hline 0.5 & 142.5 & 230 & 0.086 & 0.524 & $19-20$ \\
\hline 0.5 & 142.5 & 230 & 0.09 & 0.524 & $20-21$ \\
\hline 0.5 & 142.5 & 230 & 0.09 & 0.524 & $21-22$ \\
\hline 0.6 & 142.5 & 230 & 0.09 & 0.524 & $22-23$ \\
\hline 0.4 & 142.5 & 230 & 0.09 & 0.524 & $23-24$ \\
\hline 0.25 & 142.5 & 230 & 0.09 & 0.524 & $24-25$ \\
\hline 0.2 & 85 & 137 & 0.09 & 0.524 & $25-26$ \\
\hline 0.3 & 48 & 75 & 0.09 & 0.524 & $26-27$ \\
\hline 0.3 & 48 & 75 & 0.09 & 0.524 & $27-28$ \\
\hline 0.3 & 48 & 75 & 0.09 & 0.524 & $28-29$ \\
\hline 0.3 & 34.5 & 57 & 0.09 & 0.524 & $29-30$ \\
\hline 0.4 & 34.5 & 57 & 0.09 & 0.524 & $30-31$ \\
\hline 0.3 & 34.5 & 57 & 0.09 & 0.524 & $31-32$ \\
\hline 0.2 & 34.5 & 57 & 0.09 & 0.524 & $32-33$ \\
\hline
\end{tabular}

